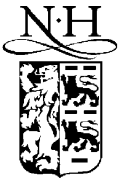

ELSEVIER

\title{
Simulations of switching vibrating cantilever in atomic force microscopy
}

\author{
M. Balantekin*, A. Atalar \\ Electrical Engineering Department, Bilkent University, Bilkent, TR-06533 Ankara, Turkey
}

Received 18 May 2002; accepted 12 August 2002

\begin{abstract}
We analyze the steady state tip sample interaction in atomic force microscopy by using an electrical circuit simulator. The phase shift between the cantilever excitation and tip, and the amplitude versus distance curves are obtained with sample stiffness as a parameter. The height shifts and hysteresis in amplitude and phase curves are observed as a result of the influence of the force between the tip and the sample. The damping and switching mechanisms are explained using the force traces obtained from simulations. The oscillation amplitude dependence of operating mode is inspected. We find that improper selection of the free tip oscillation amplitude is the cause of operating state transitions.
\end{abstract}

(C) 2002 Elsevier Science B.V. All rights reserved.

Keywords: Tip oscillation; Switching; Damping; Hysterisis

\section{Introduction}

Atomic force microscope (AFM) [1] is a powerful tool used for high resolution surface imaging. In contact mode, the tip is brought into close contact with the sample and the deflection of the tip is detected. This mode is stable and gives the highest resolution but has a drawback that the strong repulsive forces may damage soft samples and the tip. In vibrating cantilever mode, the tip oscillates at or near its resonance frequency above the surface. In this case, the amplitude or the phase of the vibrating cantilever is used for feedback. Depending on the free oscillation amplitude it is called either noncontact or intermittent contact mode. Noncontact AFM is primarily used for imaging very soft materials. One of the most important problem in this mode is that

\footnotetext{
${ }^{*}$ Corresponding author. Tel.: +90-312-290-1247. E-mail address: mujdat@ee.bilkent.edu.tr (M. Balantekin).
}

the resolution is limited to the scales on the order of the tip-sample separation [2]. Since the amplitude is small, the tip can be captured by the sample. Immersing the tip and the sample in a liquid is suggested so that the negative Hamaker constant results in repulsive van der Waals (vdW) forces which prevent the tip from jumping into contact with the sample [3]. In the tapping mode, the amplitude is larger than that of the noncontact mode such that both attractive and repulsive force regions may be entered. Although there is a behavioral description [4] of the two modes one cannot distinguish them by just considering amplitude damping in the absence of phase data. In fact, operating the microscope in one of these states is an important issue for phase imaging since the same material shows different responses for each mode.

There are several studies [5-7] discussing the dynamics of the vibrating tip. All of them reported step-like discontinuity called "switch point" in the 
amplitude versus distance curves both experimentally and theoretically. Kühle et al. [7] and Garcia and Paulo [5] reported imaging artifacts due to the transition from the low oscillation state to the high oscillation state or vice versa. Although the damping, switching and hysteresis in amplitude and phase curves are observed in experiments, a clear explanation of these phenomena does not exist. In order to interpret the experimental data correctly, we must have a good model that can be easily simulated in the time domain.

\section{Model}

In a point-mass model, the tip is modelled as a forced oscillator and the sample is represented with a spring and a damper. This results in the following pair of differential equations:

$m \ddot{z}_{\mathrm{t}}+\left(\frac{m w_{0}}{Q}\right) \dot{z}_{\mathrm{t}}+k_{\mathrm{t}}\left(z_{\mathrm{t}}-z_{\mathrm{r}}\right)-f_{\mathrm{ts}}=F_{\mathrm{d}} \cos (w t)$, $\gamma_{\mathrm{s}} \dot{z}_{\mathrm{s}}+k_{\mathrm{s}} z_{\mathrm{s}}+f_{\mathrm{ts}}=0$

where $F_{\mathrm{d}}$ and $w$ are the amplitude and angular frequency of driving force, $m, Q, w_{0}, k_{\mathrm{t}}$ are the mass, quality factor, resonance frequency and spring constant of the cantilever, respectively; $\gamma_{\mathrm{s}}$ and $k_{\mathrm{s}}$ are the damping coefficient and stiffness of the sample. The mass of the sample is ignored. The derivatives are taken with respect to time; $z_{\mathrm{r}}$ is the rest position of the tip above the sample; $z_{\mathrm{t}}$ and $z_{\mathrm{s}}$ are the positions of the tip and sample, respectively. Interaction force, $f_{\mathrm{ts}}$, can be approximated as a function of tip-to-sample distance, $d$, by [8]

$$
\begin{aligned}
& d_{0}=\sqrt[6]{\frac{\sigma}{30}}, \\
& f_{\mathrm{ts}}(d)=\frac{H R}{6 \sigma^{2}}\left[-\left(\frac{\sigma}{d}\right)^{2}+\frac{1}{30}\left(\frac{\sigma}{d}\right)^{8}\right], \quad d>d_{0},
\end{aligned}
$$

$$
f_{\mathrm{ts}}(d)=\frac{8 \sqrt{2 R}}{3\left[\left(\left(1-v_{1}^{2}\right) / E_{1}\right)+\left(\left(1-v_{2}^{2}\right) / E_{2}\right)\right]}\left(d_{0}-d\right)^{1.5},
$$

$d \leq d_{0}$,

(a)

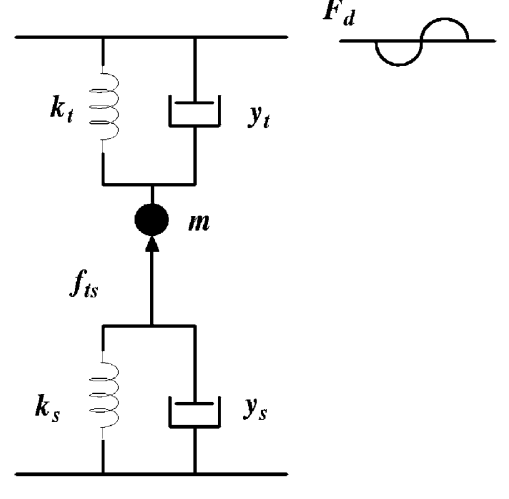

(b)

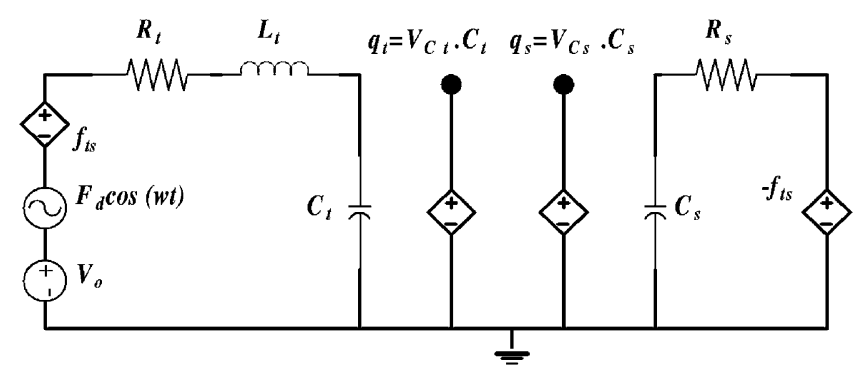

Fig. 1. (a) The mechanical model for tip-sample system; (b) equivalent electrical model; $t$ stands for tip, s stands for sample. 
where $H, R, \sigma, E_{\mathrm{i}}$, and $v_{\mathrm{i}}$ are the Hamaker constant, radius of the tip, interatomic distance, Young's modulus, and Poisson coefficient, respectively. The mechanical model is depicted in Fig. 1(a).
Recently a new method [9] is proposed to simulate the tip-sample system. One can replace masses with inductors, springs with capacitors, dampers with resistors, and forces with voltages. We can then
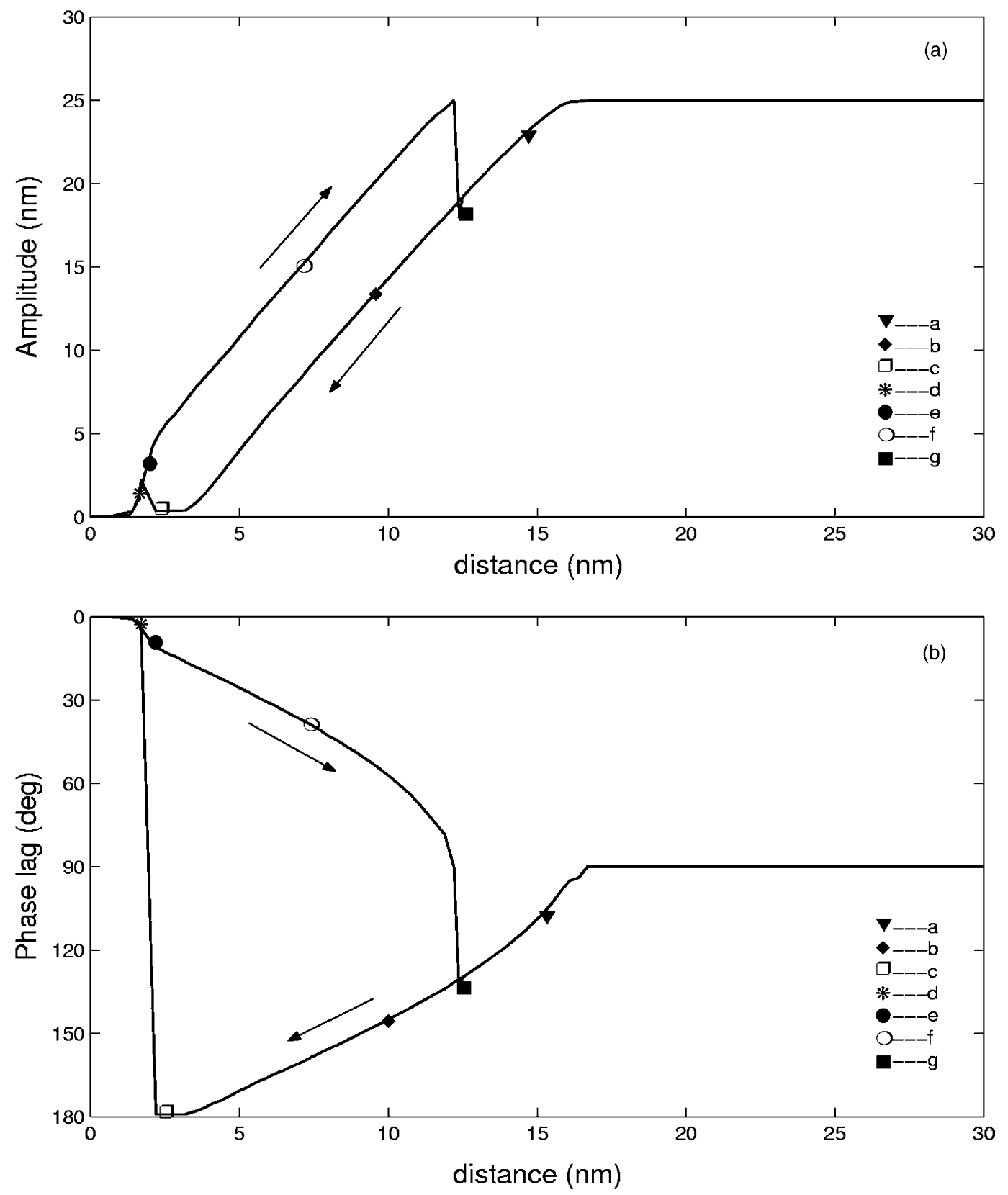

Fig. 2. Amplitude and phase vs. distance simulation results at resonance frequency for $k_{\mathrm{s}}=25 \mathrm{~N} / \mathrm{m}$ : (a) amplitude; (b) phase lag. Markers show the positions where the force traces in Fig. 3 are taken. 
(a)

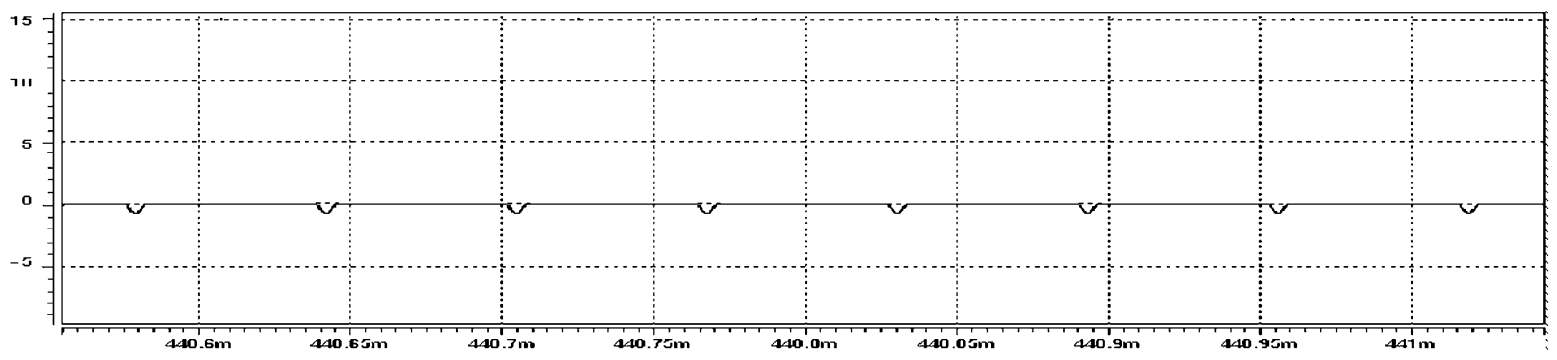

(b)

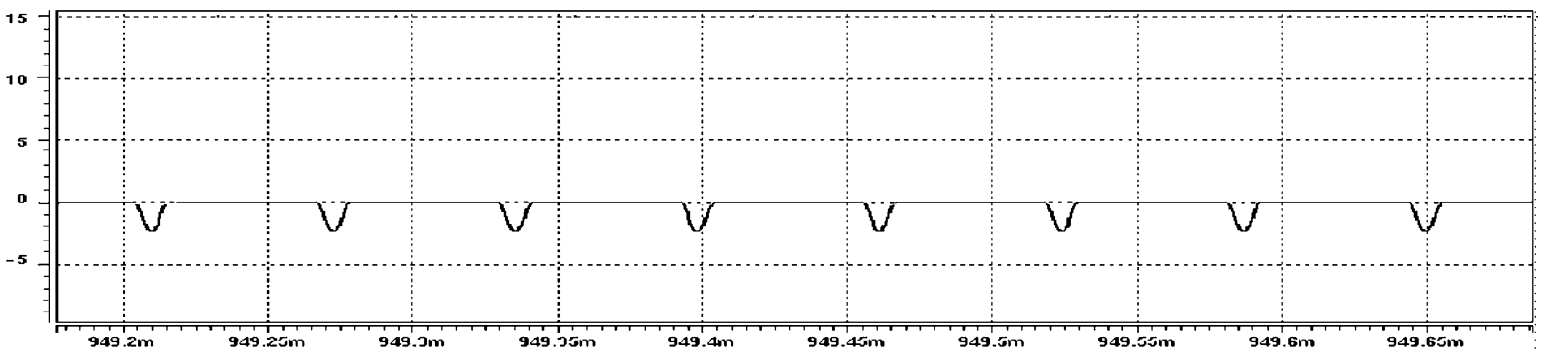

(c)

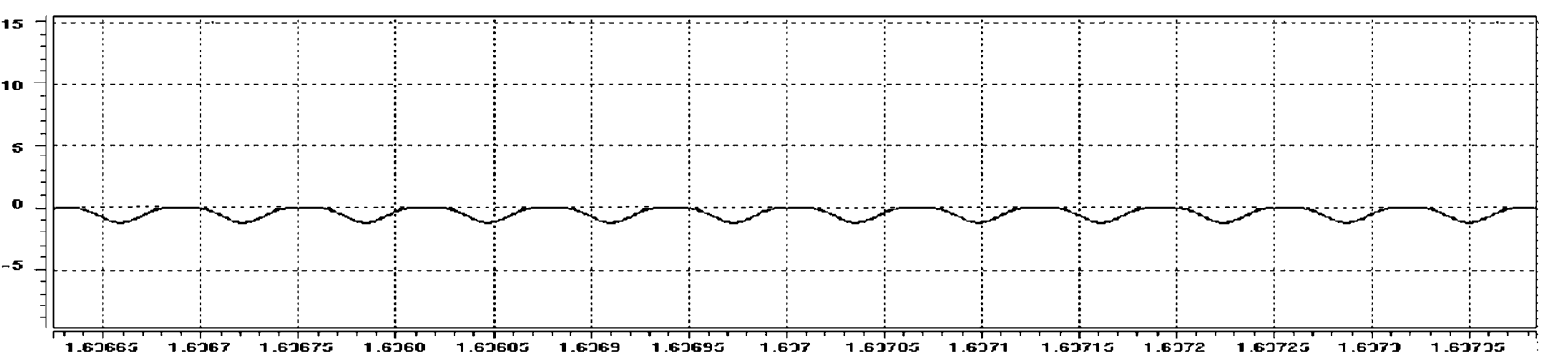

(d)

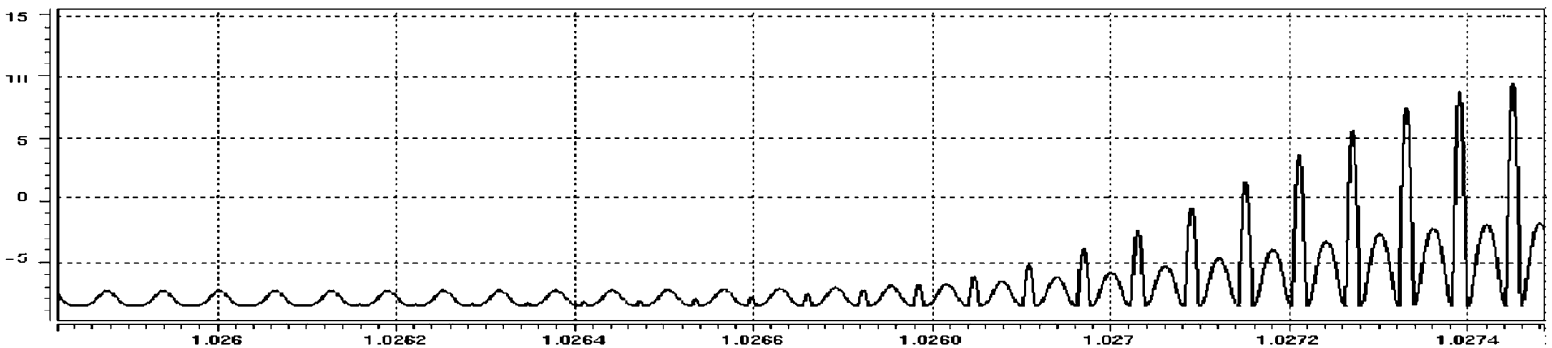

Fig. 3. Interaction forces $(\mathrm{nN})$ vs. time (s) traces taken from simulations for $k_{\mathrm{s}}=25 \mathrm{~N} / \mathrm{m}$ : (a-d) corresponds to approach cycles. The tip-tosample distance for (a) is the largest one and for (d) is the smallest; (e-g) corresponds to retract cycles. The tip-to-sample distance for (e) is the smallest one and $(\mathrm{g})$ is the largest.

rewrite the original pair of differential equations as follows:

$$
\begin{aligned}
& L_{\mathrm{t}} \ddot{q}_{\mathrm{t}}+R_{\mathrm{t}} \dot{q}_{\mathrm{t}}+\frac{q_{\mathrm{t}}}{C_{\mathrm{t}}}-f_{\mathrm{ts}}=F_{\mathrm{d}} \cos (w t)+V_{0}, \\
& R_{\mathrm{s}} \dot{q}_{\mathrm{s}}+\frac{q_{\mathrm{s}}}{C_{\mathrm{s}}}+f_{\mathrm{ts}}=0,
\end{aligned}
$$

where the charges of the capacitors, $q_{\mathrm{t}}$ and $q_{\mathrm{s}}$, represent the position of the tip and sample with respect to reference point. The equations define an equivalent electrical circuit shown in Fig. 1(b). We can simulate this circuit with a powerful electrical simulator such as $\mathrm{HSPICE}^{1}$ to find tip-sample interactions.

\footnotetext{
${ }^{1}$ HSPICE is a trademark of Meta-Software, Inc.
} 


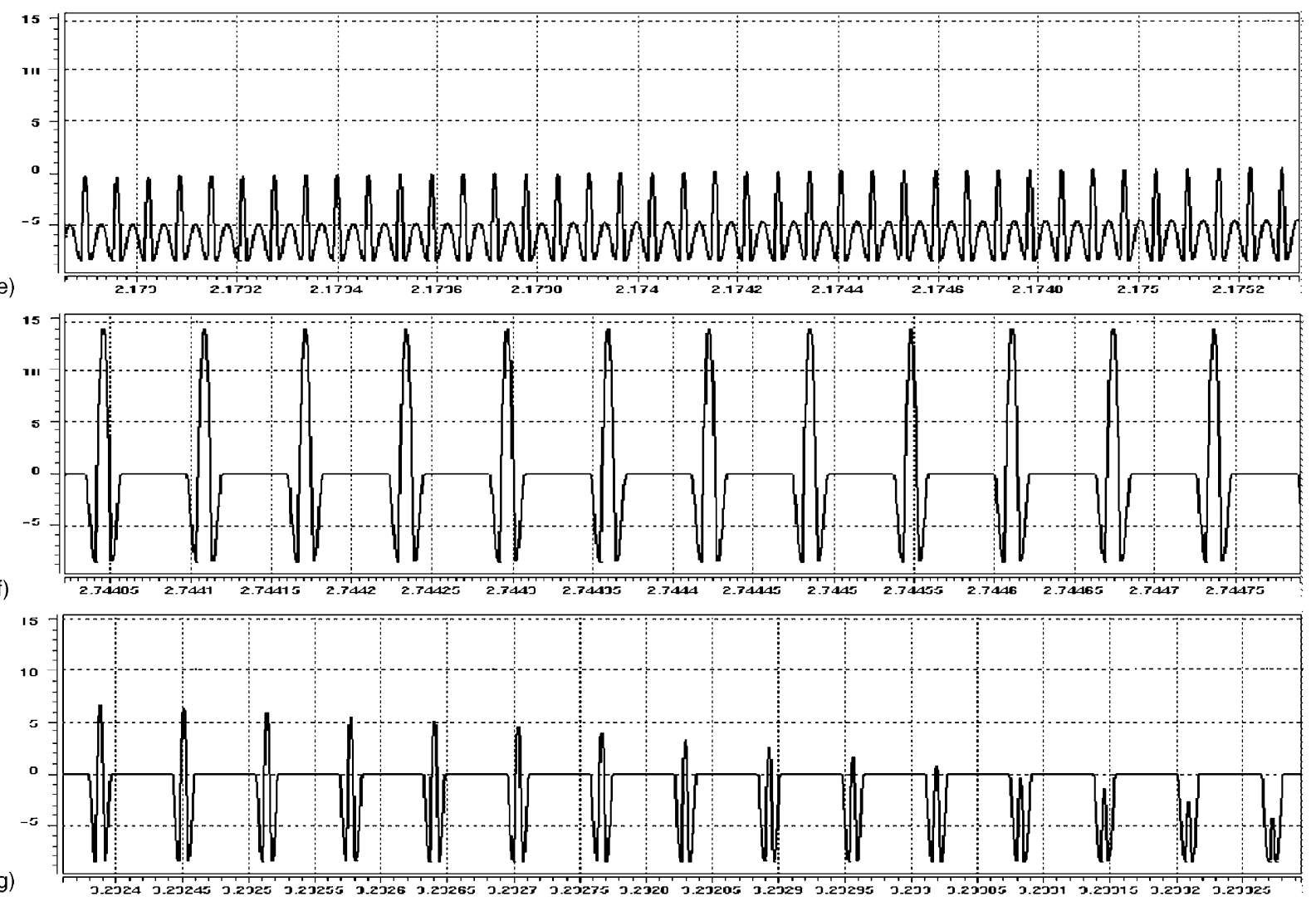

Fig. 3. (Continued).

\section{Simulations}

For the simulations, driving frequency is set to resonance frequency of the cantilever. The peak to peak free oscillation amplitude of the cantilever is $25 \mathrm{~nm}$. The cantilever parameters are chosen to be $k_{\mathrm{t}}=10 \mathrm{~N} / \mathrm{m}, Q=250, f_{0}=15.9 \mathrm{kHz}$ with $100 \mathrm{~nm}$ tip radius. The value of tip radius is chosen such that Eq. (4) is valid [10]. It is assumed that the damping constant of the sample is low and the Young's modulus of the tip is larger than that of the sample. The relation between the spring constant of a material and elastic modulus of a material and the maximum force exerted on sample is given in the literature [11]. When simulations are performed, the tip approaches or retracts from the sample with a speed of $10 \mathrm{~nm} / \mathrm{s}$.

Fig. 2 shows the amplitude and phase versus distance curve for a sample spring constant of $25 \mathrm{~N} / \mathrm{m}$.
When the tip approaches the sample, the oscillation amplitude is damped as a result of the interaction forces that exist between the tip and the sample. In the absence of the phase data, we cannot say whether it is attractive or repulsive damping. By looking at the phase difference between the tip and the driving force, we can conclude that for attractive damping where the tip is in the noncontact regime, the phase shift is more than $90^{\circ}$. While the tip is in tapping mode, the phase shift is less than $90^{\circ}$ and repulsive damping occurs.

While the tip oscillates above the sample, interaction forces are created. In order to analyze the damping mechanism, we need to find these forces. Fig. 3 shows the force traces taken from simulations for $k_{\mathrm{s}}=25 \mathrm{~N} / \mathrm{m}$. These traces together with the phase curve shown in Fig. 2(b) explain damping and switching mechanisms. Fig. 3(a)-(d) is taken when the tip approaches the sample while Fig. 3(e)-(g) correspond 


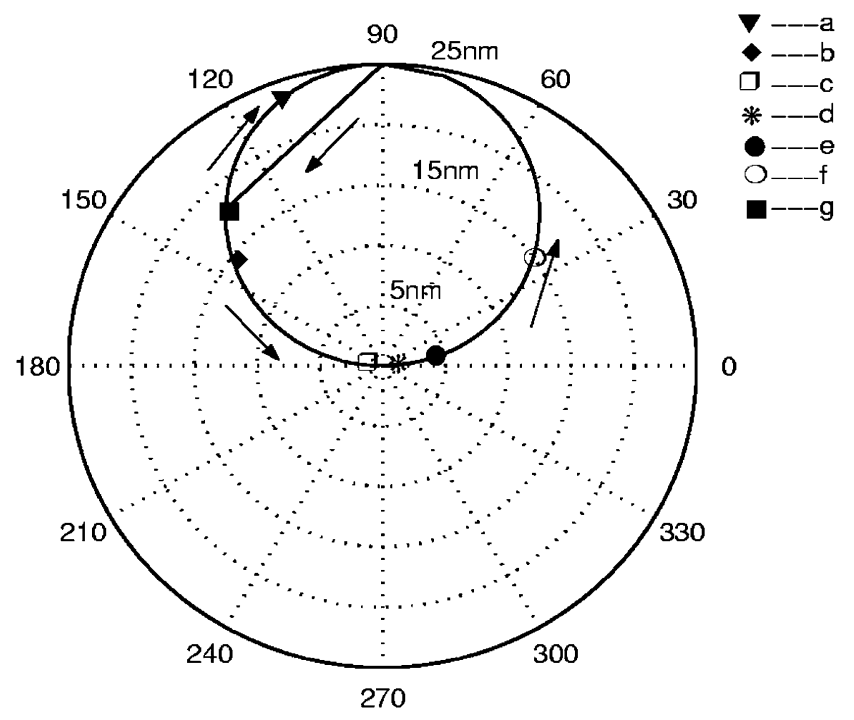

(A)

Tip oscillation (Vtip)
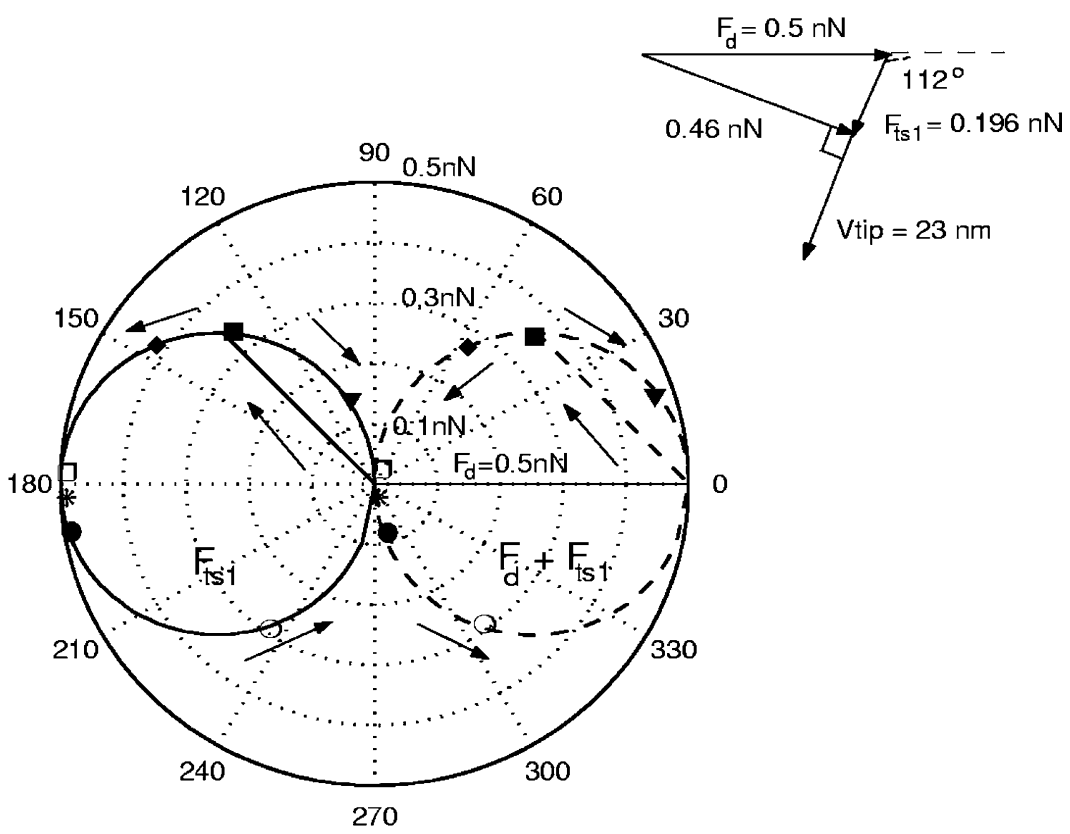

(B) First harmonic of interaction force $\left(\mathrm{F}_{\mathrm{tst}}\right)$ and total force $\left(F_{d}+F_{t s 1}\right)$

Fig. 4. Trajectories of (A) tip oscillation, (B) first harmonic of interaction force, and total force for $k_{\mathrm{s}}=25 \mathrm{~N} / \mathrm{m}$. Solid line shows $\boldsymbol{F}_{\mathrm{ts} 1}$ and dashed line shows $\boldsymbol{F}_{\mathrm{d}}+\boldsymbol{F}_{\mathrm{ts} 1}$. Each marker corresponds to same marker in Fig. 2(b). The phasor diagram shows the situation indicated with marker (a). 
to retract cycles. Their corresponding places are indicated in Fig. 2.

While the cantilever is oscillating freely (in the absence of interaction forces), there is a $90^{\circ}$ phase difference between the driving force, $\boldsymbol{F}_{\mathrm{d}}$, and tip oscillation. From now on boldface symbols represent the phasors. In Fig. 3(a), tip-to-sample distance is $15.6 \mathrm{~nm}$ and the small pulses are the attractive forces.
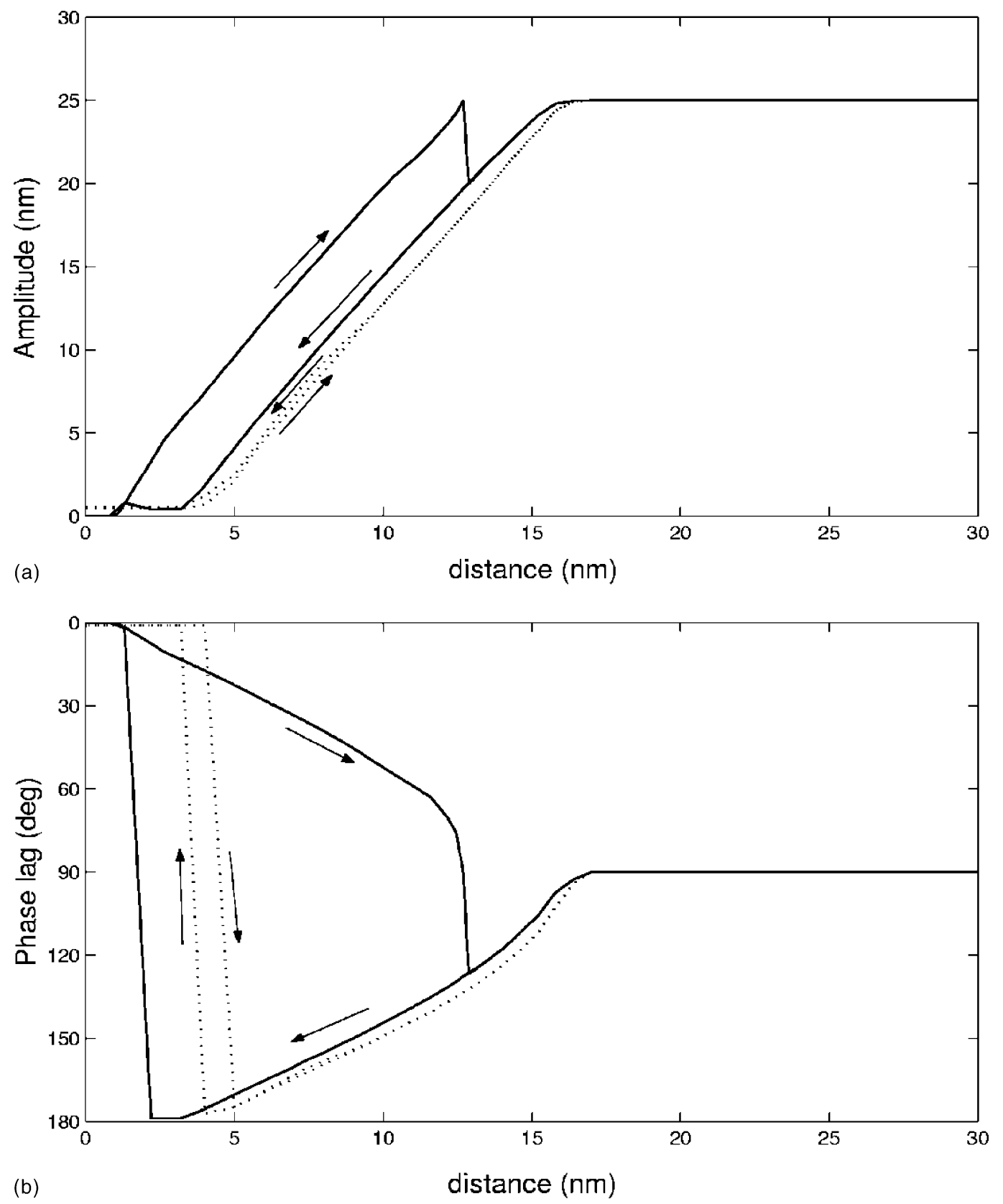

Fig. 5. Amplitude and phase vs. distance simulation results at resonance frequency for $k_{\mathrm{s}}=2.5 \mathrm{~N} / \mathrm{m}$ (dotted lines) and for $k_{\mathrm{s}}=250 \mathrm{~N} / \mathrm{m}$ (solid lines): (a) amplitude; (b) phase lag. 
If we take the Fourier series of these pulses by setting the reference point such that the tip oscillation is a cosine, then due to even symmetry, the resultant first harmonic is also a cosine. That means, the first harmonic of the force is in-phase with the tip oscillation, $\boldsymbol{V}_{\text {tip. }}$. In addition to the driving force, we now also have the first harmonic of the interaction force, $\boldsymbol{F}_{\mathrm{ts} 1}$. The combination of them must have a $90^{\circ}$ phase shift with respect to tip oscillation. These two constraints make the amplitude decrease. This situation is explained in Fig. 4 which shows the trajectories of tip oscillation, first harmonic of interaction force, and

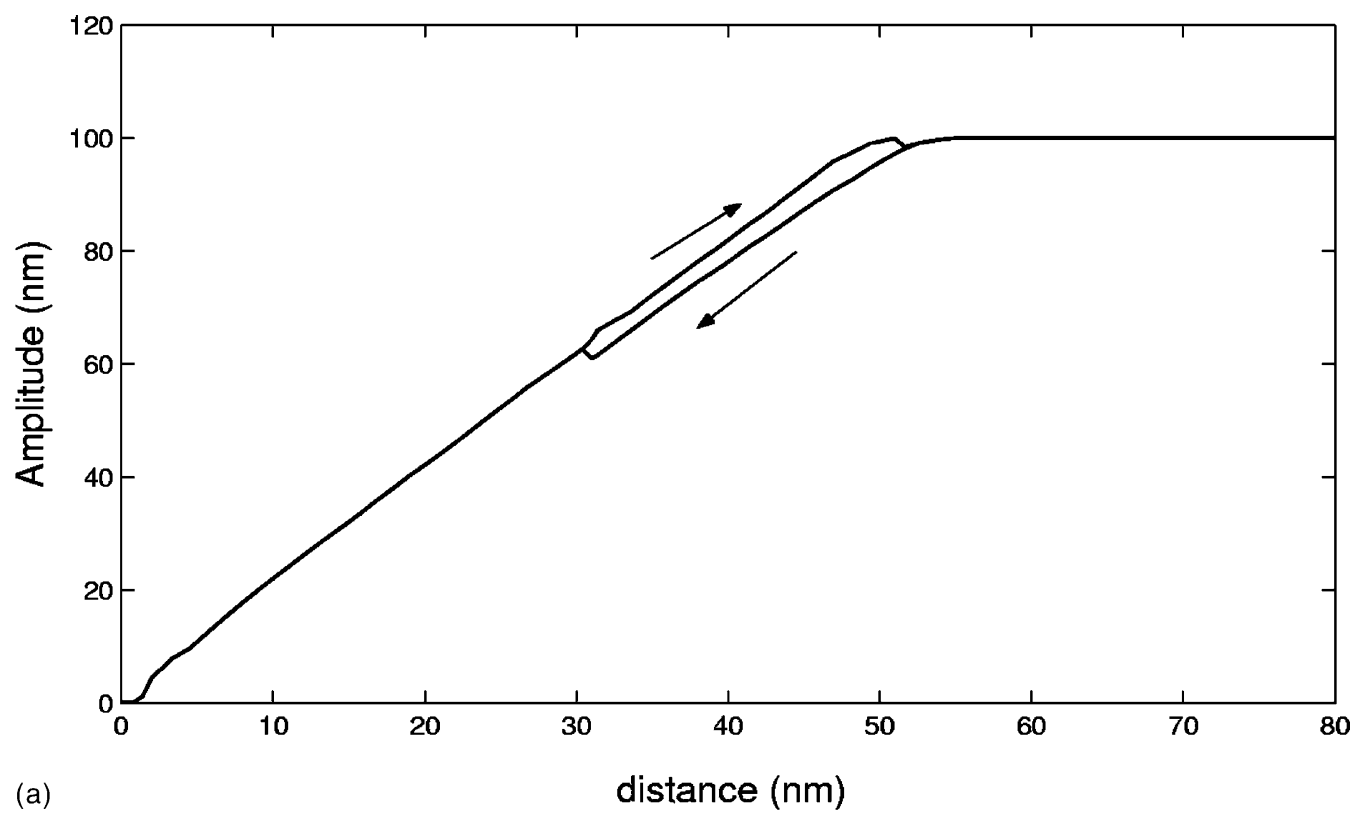

(a)

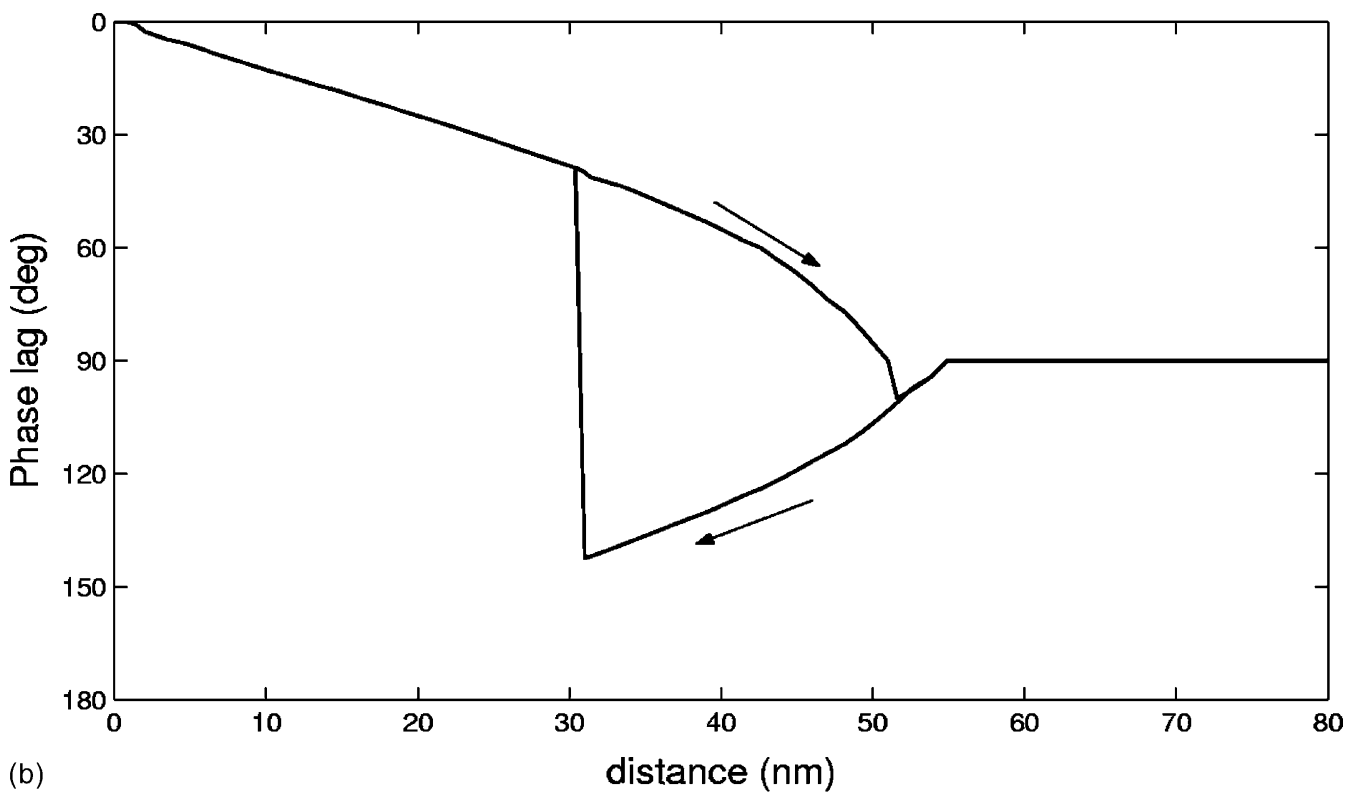

Fig. 6. Amplitude and phase vs. distance simulation results at resonance frequency for $k_{\mathrm{s}}=25 \mathrm{~N} / \mathrm{m}$ : (a) amplitude; (b) phase lag. The free tip oscillation amplitude is $100 \mathrm{~nm}$. 

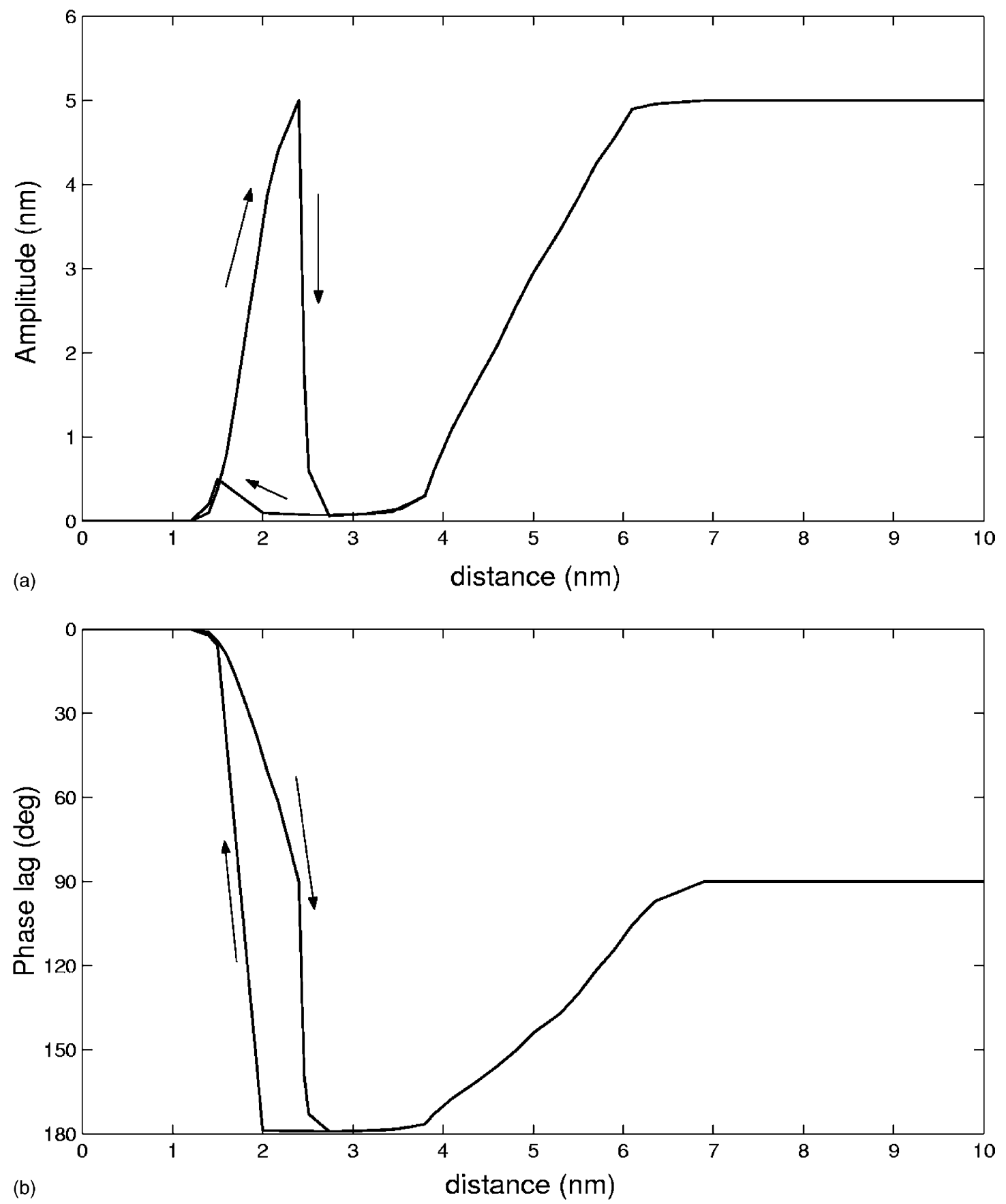

Fig. 7. Amplitude and phase vs. distance simulation results at resonance frequency for $k_{\mathrm{s}}=25 \mathrm{~N} / \mathrm{m}$ : (a) amplitude; (b) phase lag. The free tip oscillation amplitude is $5 \mathrm{~nm}$. 
total force. A phasor diagram is also given for this condition. $\boldsymbol{F}_{\mathrm{d}}$ has an amplitude of $0.5 \mathrm{nN}$ and phase of $0^{\circ}$. The markers from (a) to $(\mathrm{g})$ correspond to same markers in Fig. 2. Since the combined force has a smaller amplitude and since the tip oscillation amplitude is proportional to this force, the oscillation amplitude decreases. With a decreasing tip-to-sample spacing, the amplitude of the negative going pulses increases as shown in Fig. 3(b). If we look at Fig. 4 again, $\boldsymbol{F}_{\text {ts1 }}$ is in-phase with $\boldsymbol{V}_{\text {tip }}$ and the amplitude continues to decrease as the total force decreases. Fig. 3(c) shows the forces almost become a cosine with a $177^{\circ}$ phase shift with respect to $\boldsymbol{F}_{\mathrm{d}}$ so that the tip oscillation amplitude is very small. Fig. 3(d) shows clearly the transition from the attractive regime to the repulsive regime. Since there is a transient term that modulates this amplitude, we take the steady state value. It is assumed that the steady state is reached after 200 oscillation cycles. In Fig. 4, the marker (d) shows the situation after the transition in approach cycle. This time $\boldsymbol{F}_{\text {ts } 1}$ is out of phase with $\boldsymbol{V}_{\text {tip }}$ but the $90^{\circ}$ phase shift constraint again makes the oscillation amplitude decrease.

While retracting the tip, first there is a fast amplitude increase. If we look at Fig. 3(e), we can see that the positive going pulses crosses over the zero point which causes a first harmonic reduction. In Fig. 3(f), tip-to-sample distance is $7.4 \mathrm{~nm}$. The forces are almost even symmetric and the first harmonic is out of phase with $\boldsymbol{V}_{\text {tip }}$. Corresponding situation is indicated with marker (f) in Fig. 4. The tip oscillation amplitude continues to increase up to a critical point where $\boldsymbol{F}_{\text {ts1 }}$ becomes zero. At the critical point, a transition in retract cycle occurs. This situation is shown in Fig. 3(g). Actually, the point where $\boldsymbol{F}_{\text {ts } 1}$ becomes zero is before this time interval. The time axis is zoomed to show how transition takes place. After this switch point $\boldsymbol{F}_{\text {ts1 }}$ again becomes in-phase with $\boldsymbol{V}_{\text {tip }}$ as shown in Fig. 4. It is important to note that this switch point depends highly on the initial conditions. If the system is started to oscillate with different initial conditions its position may change.

We obtain amplitude and phase curves for different material spring constants. Fig. 5 shows two of them: one is softer $\left(k_{\mathrm{s}}=2.5 \mathrm{~N} / \mathrm{m}\right)$ than the sample we have just described and the other is stiffer $\left(k_{\mathrm{s}}=250 \mathrm{~N} / \mathrm{m}\right)$. It clearly indicates that for stiff samples we have a large hysteresis which is not desired in normal operating conditions, but may be used for differentiating two samples with different elastic properties. However, for compliant materials, we see more damping since the surface deformation is more and there is negligible hysteresis. While the material gets softer and softer, the slope of the amplitude curve increases. Hysteresis for hard surfaces is formed due to state transitions which indicate a sign change of the force gradient. This effect makes it impossible to use vibrating cantilever mode at resonant frequency for a wide range of materials. This is because of the fact that the same oscillation amplitude can be reached for two different tip-to-sample distances for any given set point amplitude. One solution to this problem is to change the oscillation frequency $[12,13]$. Another solution is to change the free tip oscillation amplitude. The simulation results for peak to peak amplitudes of 100 and $5 \mathrm{~nm}$ is given in Figs. 6 and 7, respectively. It is seen that for $100 \mathrm{~nm}$ amplitude, the tapping mode is enhanced. By selecting a proper set point amplitude, the feedback ensures the microscope to operate safely in tapping mode. So the tapping mode is more stable than noncontact mode for this material. Decreasing the oscillation amplitude is not a good choice, since it creates the possibility of switching between noncontact and tapping modes. For very soft materials tapping mode is hard to achieve since the sample surface is pulled up easily by the tip. This makes the tip amplitude vanish, and the microscope generally operates in noncontact mode. However, for relatively stiff materials, higher repulsive forces causes the tip to go into tapping state. In conclusion, for stiff materials it is better to choose high oscillation amplitudes.

\section{Conclusions}

In this paper, the amplitude and phase characteristics of oscillating tip is analyzed using an electrical circuit. The large hysteresis effect is found which explains the previously reported experimental observations. Switching and damping mechanisms are explained using force traces taken from simulations. The polar plots are given to help to understand the mechanisms involved in the nonlinear system. Effect of tip oscillation amplitude is discussed. We hope that the simulations make the experimental data interpretation more accurate. 


\section{References}

[1] G. Binning, C.F. Quate, C. Gerber, Phys. Rev. Lett. 56 (1986) 930.

[2] J.L. Hutter, J. Bechhoefer, J. Appl. Phys. 73 (1993) 4123.

[3] U. Hartmann, Phys. Rev. B 43 (1991) 2404.

[4] P.J. de Pablo, J. Colchero, M. Luna, J. Gomez-Herrero, A.M. Baro, Phys. Rev. B 61 (2000) 14179.

[5] R. Garcia, A.S. Paulo, Phys. Rev. B 61 (2000) 381.

[6] B. Anczykowski, D. Krüger, K.L. Babcock, H. Fuchs, Ultramicroscopy 66 (1996) 251.
[7] A. Kühle, A.H. Sorensen, J.B. Zandbergen, J. Bohr, Appl. Phys. A 66 (1998) S329.

[8] M. Hoummady, E. Rochat, E. Farnault, Appl. Phys. A 66 (1998) S935.

[9] O. Sahin, A. Atalar, Appl. Phys. Lett. 78 (2001) 2973.

[10] C. Argento, R.H. French, J. Appl. Phys. 80 (1996) 6081.

[11] R.G. Winkler, J.P. Spatz, S. Sheiko, M. Möller, P. Reineker, O. Marti, Phys. Rev. B 54 (1996) 8908.

[12] P. Gleyzes, P.K. Kuo, A.C. Boccara, Appl. Phys. Lett. 58 (1991) 2989.

[13] A. Kühle, A.H. Sorensen, J. Bohr, J. Appl. Phys. 81 (1997) 6562. 